\title{
Huoshanmycins A-C, New Polyketide Dimers Produced by Endophytic Streptomyces sp. HS-3-L-1 From Dendrobium huoshanense
}

\author{
Youjuan Zhu ${ }^{1 \dagger}$, Yichao Kong ${ }^{2 \dagger}$, Yu Hong ${ }^{1}$, Ling Zhang ${ }^{1}$, Simin $\mathrm{Li}^{1}$, Shurong $\mathrm{Hou}^{2}$, \\ Xiabin Chen ${ }^{2}$, Tian $\mathrm{Xie}^{2}$, Yang $\mathrm{Hu}^{1 *}$ and Xiachang Wang ${ }^{1 *}$ \\ ${ }^{1}$ Jiangsu Key Laboratory for Functional Substances of Chinese Medicine, Nanjing University of Chinese Medicine, Nanjing, China, \\ ${ }^{2}$ Key Laboratory of Element Class Anti-Cancer Chinese Medicines, Engineering Laboratory of Development and Application of \\ Traditional Chinese Medicines, Collaborative Innovation Center of Traditional Chinese Medicines of Zhejiang Province, School of \\ Pharmacy, Hangzhou Normal University, Hangzhou, China
}

Three new polyketide dimers named huoshanmycins A-C (1-3) were isolated from a plant endophytic Streptomyces sp. HS-3-L-1 in the leaf of Dendrobium huoshanense, which was collected from the Cultivation base in Jiuxianzun Huoshanshihu Co., Ltd. The dimeric structures of huoshanmycins were composed of unusual polyketides SEK43, SEK15, or UWM4, with a unique methylene linkage. Their structures were elucidated through comprehensive 1D-/2D-NMR and HRESIMS spectroscopic data analysis. The cytotoxicity against MV4-11 human leukemia cell by the Cell Counting Kit-8 (CCK8) method was evaluated using isolated compounds with triptolide as positive control $\left(\mathrm{C}_{50}: 1.1 \pm 0.4 \mu \mathrm{M}\right)$. Huoshanmycins $\mathrm{A}$ and $\mathrm{B}(\mathbf{1}, \mathbf{2})$ displayed moderate cytotoxicity with $\mathrm{IC}_{50}$ values of $32.9 \pm 7.2$ and $33.2 \pm 6.1 \mu \mathrm{M}$, respectively.

Keywords: huoshanmycin, polyketide, Streptomyces, Dendrobium huoshanense, endophyte

\section{INTRODUCTION}

Dendrobium huoshanense is a perennial epiphytic Orchidaceae herb with important medicinal and ornamental value. The leaves of $D$. huoshanense have long been utilized for dermatologic disorders, metabolic syndromes, nervous system disorders, and musculoskeletal system disorders (Wang, 2021). Modern pharmacological research has revealed that D. huoshanense has anti-inflammatory (Ge et al., 2018; Li et al., 2020), cytotoxic (Chen et al., 2022), hypoglycemic (Wang et al., 2019), antiatherosclerosis (Fan et al., 2020), and antioxidant (Tian et al., 2013) activity. Dendrobium plant is well-known for its rich and diverse endophytic bacterial and fungal community (Chen et al., 2019; Chen et al., 2020). Previous studies have revealed the close relationship between Dendrobium and its endophytes, such as improving the seed germination rate (Tsavkelova et al., 2007) and supply of nutrients (Li et al., 2017). At present, research on D. huoshanense endophytes mainly focuses on the diversity and functions, while not much is known about their secondary metabolites, especially for bacteria. The main species of bacterial microorganisms of D. huoshanense are Sphingomonas, Acinetobacter, Enterococcus, Bacillus, and Methylobacterium (Chen et al., 2020). Streptomyces is the largest genus of Actinobacteria and characterized by producing complex secondary metabolites. They produce over two-thirds of the clinically useful antibiotics of natural origin (e.g., chloramphenicol, streptomycin, tetracycline, erythromycin, ivermectin, and rifamycin) (Raja and Prabakarana, 2011). The last four compounds all belong to polyketides, which are derived from a 
precursor molecule consisting of a chain of alternating ketone (or reduced forms of a ketone) and methylene groups.

Since the discovery of taxol and taxane produced by an endophytic fungus from the phloem (inner bark) of Pacific yew in 1993 (Stierle et al., 1993), endophytes have become an important resource in the field of bioactive natural products discovery (Newman and Cragg, 2015; Gómez and Luiz, 2018), as they can produce analogs or bioactivity-related compounds as their hosts did (Cui et al., 2012; Zhao et al., 2020c). As part of an effort to characterize novel natural products from medicinal plants (Wang et al., 2009; Ding et al., 2021; Hu et al., 2021) and their endophytes (Zhao et al., 2020b; Zhao et al., 2020a; Zhu et al., 2021), herein we report the isolation and characterization of three new polyketide dimers from an endophyte Streptomyces sp. HS-3-L-1 of the D. huoshanense leaf. The dimeric structures of new huoshanmycins A-C (1-3) were composed of SEK43, SEK15, or UWM4 (Meurer et al., 1997), with a unique methylene linkage. Herein, we report the fermentation, extraction, isolation, structural elucidation, and cytotoxic activity of these secondary metabolites.

\section{MATERIALS AND METHODS}

\section{General Experimental Procedures}

UV data were acquired on a Persee TU-1810 spectrophotometer (Persee analytics, Beijing, China). IR spectra were measured on a Thermo Scientific Nicolet iS5 FT-IR spectrometer (Thermo, United States). NMR spectra were obtained on a Bruker Advance AV500 spectrometer (Bruker, Germany). HRESIMS spectra were recorded on an Orbitrap Elite mass spectrometer (Thermo Scientific, United States). Liquid chromatography-mass spectrometry (LC-MS) was conducted with an Agilent 1290 system equipped with 6120 Quadrupole MSD mass spectrometer (Agilent Technologies, United States). HPLC analysis was performed on a Waters 2695 system equipped with 2998 PDA detector. Total component analysis was performed on an Agilent 1290 UHPLC-6520 Q-TOF/MS. Preparative HPLC separation was performed on a Waters 1525 EF LC system (Waters Company, United States). MCI GEL high-porous polymer $(75-150 \mu \mathrm{m})$ was purchased from Mitsubishi Chemical Corporation (Japan). Sephadex LH-20 resin $(25-100 \mu \mathrm{m})$ was purchased from GE Healthcare Company (Sweden). XAD16N resin (20-60 mesh) was obtained from Yuanye Company (Nanjing, Jiangsu, China). Chemicals were purchased from Juyou Company or Aldrich and used without further purification unless otherwise noted.

\section{Strain Isolation}

Plant samples of $D$. huoshanense were provided by Jiuxianzun Huoshanshihu Co., Ltd. (Liu-An City, Anhui Province, China) and identified by co-author Dr. Yang Hu. A voucher specimen (no. 20190309) was deposited at Jiangsu Key Laboratory for Functional Substances of Chinese Medicine, China. The roots, leaves, and stems of $D$. huoshanense were separated and cleaned with water and then rinsed in $0.1 \%$ Tween-20 for $30 \mathrm{~s}$, sequentially immersed in $75 \%$ ethanol for $5 \mathrm{~min}$ and in $2 \%$ sodium hypochlorite for $5 \mathrm{~min}$ and rinsed with $10 \% \mathrm{NaHCO}_{3}$ for $10 \mathrm{~min}$ to inhibit fungal growth. After each treatment, samples were rinsed three times in sterile water. The surface sterilized samples were aseptically dissected into small pieces; $0.5 \mathrm{~g}$ of each sample was suspended in $1.0 \mathrm{ml}$ of sterile $\mathrm{H}_{2} \mathrm{O}$, and heated at $75^{\circ} \mathrm{C}$ for $1 \mathrm{~min}$ to eliminate nonsporulating bacteria (Zhao et al., 2020a). A $100-\mu \mathrm{l}$ aliquot of supernatant was streaked on oatmeal agar and on ISP4 agar plates supplemented with nalidixic acid $(25 \mu \mathrm{g} / \mathrm{ml})$ and amphotericin B $(25 \mu \mathrm{g} / \mathrm{ml})$. A number of sporulating bacterial colonies were observed after 1-2 months of incubation at $28^{\circ} \mathrm{C}$, and each colony was subsequently purified on a M2 agar plate (Wang et al., 2013). Overall, 54 endophytic strains were isolated from plant samples. The endophytic strain HS-3-L-1 was isolated from the leaf of $D$. huoshanense.

\section{Phylogenetic Analysis}

Strain HS-3-L-1 was inoculated in a $20-\mathrm{ml}$ test tube with $4 \mathrm{ml}$ of TSB broth. After 3 days culture at $28^{\circ} \mathrm{C}$ with $160 \mathrm{rpm}$ agitation, the partial 16S rRNA gene fragment was amplified using universal primers (27F 5'-AGAGTTTGATCMTGGCTCAG-3'; 1492R 5' GGTTACCTTGTTACGACTT- $3^{\prime}$ ). The amplified fragment ( $1,367 \mathrm{bp}$ ) was sent for sequencing analysis (Shanghai Sangong Company, China), which displayed $99.85 \%$ identity (BlastN, https://blast.ncbi.nlm.nih.gov/Blast.cgi) to Streptomyces polaris (MW164959.1). The sequence of 16S rRNA has been deposited in the NCBI nucleotide database with the accession number OK161010.

\section{Fermentation, Extraction, and Isolation}

Streptomyces sp. HS-3-L-1 was grown on M2 agar plate (glucose $4 \mathrm{~g} / \mathrm{L}$, malt extract $10 \mathrm{~g} / \mathrm{L}$, yeast extract $4 \mathrm{~g} / \mathrm{L}$, and agar $15 \mathrm{~g} / \mathrm{L}$ ) at $28^{\circ} \mathrm{C}$ for a week. Small pieces of agar with bacterial growth were added to eleven 250-ml Erlenmeyer flasks, each containing $50 \mathrm{ml}$ of medium Bran [corn flour, $40.0 \mathrm{~g} / \mathrm{L}$; gluten powder, $5.0 \mathrm{~g} / \mathrm{L}$; $\mathrm{K}_{2} \mathrm{HPO}_{4} \bullet 3 \mathrm{H}_{2} \mathrm{O}, 0.5 \mathrm{~g} / \mathrm{L}$; glucose, $10.0 \mathrm{~g} / \mathrm{L}$; bran, $10.0 \mathrm{~g} / \mathrm{L} ; \mathrm{CaCO}_{3}$, $2.0 \mathrm{~g} / \mathrm{L}$; and $\left.\left(\mathrm{NH}_{4}\right)_{2} \mathrm{SO}_{4}, 1.0 \mathrm{~g} / \mathrm{L}\right]$. After 3 days of incubation at $28^{\circ} \mathrm{C}$ with $200 \mathrm{rpm}$ agitation, the seed cultures were used to inoculate 100 Erlenmeyer flasks $(250 \mathrm{ml})$, each containing $100 \mathrm{ml}$ of medium Bran (total $10 \mathrm{~L}$ ). The fermentation was carried out on a rotary shaker $(200 \mathrm{rpm})$ at $28^{\circ} \mathrm{C}$ for a week. All obtained culture broth was combined and centrifuged at 5,000 $\times g$ for $30 \mathrm{~min}$ to separate the mycelium and supernatant. Mycelium was extracted with $\mathrm{MeOH}(3 \times 2 \mathrm{~L})$, and the organic phase was evaporated to afford $50.2 \mathrm{~g}$ of crude extract A. The supernatant was mixed with $4 \%(\mathrm{w} / \mathrm{v})$ XAD-16 resin and stirred for $6 \mathrm{~h}$, followed by filtration. The resin was washed with water $(3 \times 500 \mathrm{ml})$ and then eluted with $\mathrm{MeOH}$ until the eluant was colorless. The $\mathrm{MeOH}$ extract was subsequently evaporated to afford $13.3 \mathrm{~g}$ of crude extract $\mathrm{B}$.

Crude extract A (50.2 g) was subjected to an MCI column $(500 \mathrm{~g}, 10 \times 80 \mathrm{~cm})$ and eluted with a gradient of aqueous $\mathrm{MeOH}$ $(20,40,60,80$, and $100 \%)$ to yield 16 fractions (Fr. 1-1 to Fr. 116). Fr. 1-9 (0.5 g) was subjected to a Sephadex LH-20 column (4 $\times 100 \mathrm{~cm}, 2 \mathrm{ml} / \mathrm{min}$ ) eluted with $80 \%$ aqueous $\mathrm{MeOH}$ to obtain 11 subfractions (Fr. 1-9-1 to Fr. 1-9-11). Compound 5 (13.6 mg) was obtained from Fr. 1-9-4. Fr. 1-9-9 was further purified by 
TABLE 1 | ${ }^{1} \mathrm{H}(500 \mathrm{MHz})$ and ${ }^{13} \mathrm{C}(125 \mathrm{MHz})$ NMR data of compounds 1-3 in DMSO- $d_{6}$.

\begin{tabular}{|c|c|c|c|c|c|c|}
\hline \multirow[t]{2}{*}{ No } & \multicolumn{2}{|c|}{1} & \multicolumn{2}{|c|}{2} & \multicolumn{2}{|c|}{3} \\
\hline & $\delta_{\mathrm{C}}$, type & $\begin{array}{l}\delta_{\mathrm{H}}, J \\
\text { in } \mathrm{Hz}\end{array}$ & $\delta_{\mathrm{C}}$, type & $\begin{array}{l}\delta_{\mathrm{H}}, J \\
\text { in } \mathrm{Hz}\end{array}$ & $\delta_{\mathrm{C}}$, type & $\begin{array}{c}\delta_{\mathrm{H}, J} \\
\text { in } \mathrm{Hz}\end{array}$ \\
\hline 1 & $165.2, \mathrm{C}$ & - & $165.2, \mathrm{C}$ & - & $165.2, \mathrm{C}$ & - \\
\hline 2 & 100.0, C & - & 100.1, C & - & 100.1, C & - \\
\hline 3 & 166.1, C & - & 166.1, C & - & 166.3, C & - \\
\hline 4 & 101.8, CH & $5.76, \mathrm{~s}$ & 101.8, CH & $5.75, \mathrm{~s}$ & 101.6, $\mathrm{CH}$ & $5.74, \mathrm{~s}$ \\
\hline 5 & $160.9, \mathrm{C}$ & - & 160.9, C & - & $160.8, \mathrm{C}$ & - \\
\hline 6 & $36.5, \mathrm{CH}_{2}$ & $3.54, \mathrm{~s}$ & 36.6, $\mathrm{CH}_{2}$ & $3.55, \mathrm{~s}$ & $36.5, \mathrm{CH}_{2}$ & $3.53, \mathrm{~s}$ \\
\hline 7 & 133.2, C & - & 133.2, C & - & 133.2, C & - \\
\hline 8 & 121.3, $\mathrm{CH}$ & $6.74, \mathrm{dd}(7.6,2.1)$ & 121.3, $\mathrm{CH}$ & $6.75, d(7.6)$ & $121.2, \mathrm{CH}$ & $6.75, \mathrm{~d}(7.7)$ \\
\hline 9 & 130.5, CH & 7.22, t (7.7) & $130.5, \mathrm{CH}$ & 7.22, t (7.9) & $130.5, \mathrm{CH}$ & $7.21, \mathrm{t}(7.9)$ \\
\hline 10 & 115.0, $\mathrm{CH}$ & $6.80, \mathrm{t}(8.7)$ & 115.0, $\mathrm{CH}$ & $6.80, d(8.2)$ & 114.9, $\mathrm{CH}$ & $6.80, d(8.2)$ \\
\hline 11 & $154.2, \mathrm{C}$ & - & 154.2, C & - & 154.2, C & - \\
\hline 12 & 131.2, C & - & 131.3, C & - & 131.2, C & - \\
\hline 13 & $200.4, C$ & - & $200.4, C$ & - & 200.6, C & - \\
\hline 14 & 116.0, C & - & 116.0, C & - & 116.0, C & - \\
\hline 15 & $165.5, \mathrm{C}$ & - & $165.5, \mathrm{C}$ & - & $165.5, \mathrm{C}$ & - \\
\hline 16 & 101.1, CH & $6.13, d(2.3)$ & 101.8, CH & $6.14, \mathrm{~s}$ & 101.1, $\mathrm{CH}$ & $6.13, d(2.1)$ \\
\hline 17 & 163.7, C & - & 163.6, C & - & 163.6, C & - \\
\hline 18 & 112.0, $\mathrm{CH}$ & $6.09, \mathrm{~d}(2.0)$ & 112.0, $\mathrm{CH}$ & $6.09, \mathrm{~s}$ & 112.0, $\mathrm{CH}$ & $6.09, \mathrm{~d}(1.7)$ \\
\hline 19 & $143.4, \mathrm{C}$ & & $143.4, \mathrm{C}$ & & $143.4, \mathrm{C}$ & \\
\hline 20 & $21.9, \mathrm{CH}_{3}$ & $1.83, \mathrm{~s}$ & $21.9, \mathrm{CH}_{3}$ & $1.84, \mathrm{~s}$ & $21.9, \mathrm{CH}_{3}$ & $1.84, \mathrm{~s}$ \\
\hline $1^{\prime}$ & $161.9, \mathrm{C}$ & - & 165.2, C & - & 165.1, C & - \\
\hline $2^{\prime}$ & 100.1, C & - & 100.1, C & - & 100.0, C & - \\
\hline $3^{\prime}$ & 166.3, C & - & 166.1, C & - & 166.2, C & - \\
\hline $4^{\prime}$ & 101.7, CH & $5.70, \mathrm{~s}$ & 101.8, CH & $5.75, \mathrm{~s}$ & 101.5, CH & $5.74, \mathrm{~s}$ \\
\hline $5^{\prime}$ & $161.4, \mathrm{C}$ & - & 160.9, C & - & 161.2, C & - \\
\hline $6^{\prime}$ & 37.0, $\mathrm{CH}_{2}$ & $3.47, \mathrm{~s}$ & 36.6, $\mathrm{CH}_{2}$ & $3.55, \mathrm{~s}$ & 36.7, $\mathrm{CH}_{2}$ & $3.48, \mathrm{~s}$ \\
\hline $7^{\prime}$ & 134.8, C & - & 133.2, C & - & 135.8, C & - \\
\hline $8^{\prime}$ & 120.9, CH & $6.74, \mathrm{dd}(7.6,2.1)$ & 121.3, $\mathrm{CH}$ & $6.75, d(7.6)$ & $109.5, \mathrm{CH}$ & $6.16, d(1.9)$ \\
\hline $9^{\prime}$ & 129.1, CH & 7.17, t (7.9) & 130.5, $\mathrm{CH}$ & $7.22, \mathrm{t}(7.9)$ & 160.1, C & \\
\hline $10^{\prime}$ & $114.4, \mathrm{CH}$ & $6.80, \mathrm{t}(8.7)$ & 115.0, $\mathrm{CH}$ & $6.80, d(8.2)$ & 101.9, $\mathrm{CH}$ & $6.23, \mathrm{~d}(1.9)$ \\
\hline $11^{\prime}$ & $154.7, \mathrm{C}$ & - & 154.2, C & - & $157.8, \mathrm{C}$ & - \\
\hline $12^{\prime}$ & 129.6, C & - & 131.3, C & - & $121.5, \mathrm{C}$ & - \\
\hline $13^{\prime}$ & 141.1, C & - & $200.4, C$ & - & 200.3, C & - \\
\hline $14^{\prime}$ & 111.3, $\mathrm{CH}$ & $5.95, d(2.4)$ & 116.0, C & - & 117.6, C & - \\
\hline $15^{\prime}$ & $162.4, \mathrm{C}$ & - & $165.5, \mathrm{C}$ & - & 163.2, C & - \\
\hline $16^{\prime}$ & 102.3, CH & $6.27, \mathrm{~d}(2.4)$ & 101.8, CH & $6.14, \mathrm{~s}$ & $100.9, \mathrm{CH}$ & $6.11, \mathrm{~s}$ \\
\hline $17^{\prime}$ & 163.6, C & - & 163.6, C & - & $162.4, \mathrm{C}$ & - \\
\hline $18^{\prime}$ & 115.9, C & - & 112.0, CH & $6.09, \mathrm{~s}$ & 111.3, $\mathrm{CH}$ & $6.06, \mathrm{~s}$ \\
\hline $19^{\prime}$ & 203.6, C & - & 143.4, C & - & 142.1, C & - \\
\hline $20^{\prime}$ & $30.3, \mathrm{CH}_{3}$ & $1.87, \mathrm{~s}$ & $21.9, \mathrm{CH}_{3}$ & $1.84, \mathrm{~s}$ & $21.4, \mathrm{CH}_{3}$ & $1.83, \mathrm{~s}$ \\
\hline $1^{\prime \prime}$ & 17.8, $\mathrm{CH}_{2}$ & $3.25, \mathrm{~s}$ & 17.8, $\mathrm{CH}_{2}$ & $3.24, s$ & 17.7, $\mathrm{CH}_{2}$ & $3.24, \mathrm{~s}$ \\
\hline $11-\mathrm{OH}$ & - & $9.81, \mathrm{~s}$ & - & $9.82, \mathrm{~s}$ & - & $9.83, \mathrm{~s}$ \\
\hline $15-\mathrm{OH}$ & - & $12.66, \mathrm{~s}$ & - & $12.68, \mathrm{~s}$ & - & $12.68, \mathrm{~s}$ \\
\hline $17-\mathrm{OH}$ & - & $10.44, s$ & - & $10.45, \mathrm{~s}$ & - & $10.45, \mathrm{~s}$ \\
\hline $9^{\prime}-\mathrm{OH}$ & - & - & - & - & - & $9.88, \mathrm{~s}$ \\
\hline $11^{\prime}-\mathrm{OH}$ & - & $9.48, \mathrm{~s}$ & - & $9.82, \mathrm{~s}$ & - & $10.26, s$ \\
\hline $15^{\prime}-\mathrm{OH}$ & - & $10.36, \mathrm{~s}$ & - & $12.68, \mathrm{~s}$ & - & $12.05, \mathrm{~s}$ \\
\hline $17^{\prime}-\mathrm{OH}$ & - & $12.50, \mathrm{~s}$ & - & $10.45, \mathrm{~s}$ & - & $10.21, \mathrm{~s}$ \\
\hline
\end{tabular}

semi-preparative HPLC (38\% aqueous $\mathrm{MeOH}$ ) to yield compound $3(5.8 \mathrm{mg})$. Crude extract B $(13.3 \mathrm{~g})$ was subjected to an MCI column $(200 \mathrm{~g}, 6 \times 30 \mathrm{~cm})$, using gradient elution from $20 \%$ to $100 \%$ aqueous $\mathrm{MeOH}$ to provide 11 fractions (Fr. 2-1 to Fr. 2-11). Fractions Fr. 2-5 (0.7 g) was subjected to a Sephadex LH-20 column $(4 \times 100 \mathrm{~cm}, 2 \mathrm{ml} / \mathrm{min}, 80 \%$ aqueous $\mathrm{MeOH})$ to obtain 12 subfractions (Fr. 2-5-1 to Fr. 2-5-12). Fr. 2-5-6 (0.3 g) was further purified by semi-preparative HPLC (45\% aqueous $\mathrm{MeOH})$ to yield compounds $4(7.6 \mathrm{mg})$ and $7(9.4 \mathrm{mg})$. Fr. 2-10 $(0.5 \mathrm{~g})$ was also purified by a Sephadex LH-20 column $(4 \times$
$100 \mathrm{~cm}, 2 \mathrm{ml} / \mathrm{min}, 80 \%$ aqueous $\mathrm{MeOH})$ to obtain seven subfractions (Fr. 2-10-1 to Fr. 2-10-7). Fr. 2-10-6 (0.2 g) was further purified by semi-preparative HPLC (63\% aqueous $\mathrm{MeOH})$ to yield compounds 1 (8.6 mg), $2(16.8 \mathrm{mg})$, and 6 (12.3 mg).

Huoshanmycin A (1): Yellow amorphous powder; UV $(\mathrm{MeOH}) \lambda_{\max }(\log \varepsilon) 294 \mathrm{~nm}$ (4.17); IR (KBr) $v_{\max }$ 3,164, $2,927,1,680,1,617,1,437,1,401,1,384,1,284,1,207,1,138$, 1,027, 829, 803, and $724 \mathrm{~cm}^{-1} ;{ }^{13} \mathrm{C}$ and ${ }^{1} \mathrm{H}$ NMR data, see

Table 1; (+)-ESI-MS: $m / z 749.0[\mathrm{M}+\mathrm{H}]^{+}$; (-)-ESI-MS: $\mathrm{m} / \mathrm{z}$ 
TABLE 2 | Antiproliferative activity of compounds 1-4 against MV4-11 cell line.

\section{Compounds}

1

2

3

Triptolide
$I_{50} \pm S D, \mu M$

$32.9 \pm 7.2$

$33.2 \pm 6.1$

$>50$

$>50$

$1.1 \pm 0.4$
$747.2[\mathrm{M} \mathrm{-} \mathrm{H}]^{-}$; (-)-HR-ESI-MS $m / z 747.17096[\mathrm{M} \mathrm{-} \mathrm{H}]^{-}($calcd. for $\mathrm{C}_{41} \mathrm{H}_{31} \mathrm{O}_{14}$ 747.1714).

Huoshanmycin B (2): Yellow amorphous powder; UV $(\mathrm{MeOH}) \lambda_{\max }(\log \varepsilon) 294 \mathrm{~nm}$ (4.44); IR (KBr) $v_{\max } 3,184$, $2,260,2,129,1,675,1,587,1,464,1,284,1,167,1,158,1,024$, $995,926,826,769,722,699,656,633,611,576$, and $523 \mathrm{~cm}^{-1}$; ${ }^{13} \mathrm{C}$ and ${ }^{1} \mathrm{H}$ NMR data, see Table 1; (+)-ESI-MS: $m / z 749.0[\mathrm{M}+$ $\mathrm{H}]^{+}$; (-)-ESI-MS: $m / z$ 747.2 $[\mathrm{M}-\mathrm{H}]^{-} ;(+)$-HR-ESI-MS $\mathrm{m} / z$ $749.18768[\mathrm{M}+\mathrm{H}]^{+}$(calcd. for $\mathrm{C}_{41} \mathrm{H}_{33} \mathrm{O}_{14}$ 749.1970).

Huoshanmycin C (3): Yellow amorphous powder; UV $(\mathrm{MeOH}) \lambda_{\max }(\log \varepsilon) 294 \mathrm{~nm}$ (4.41); IR (KBr) $v_{\max } 3,172$, $2,259,2,129,1,677,1,616,1,587,1,464,1,384,1,271,1,204$, $1,169,1,142,1,024,999,926,844,800,766,722,600$, and $524 \mathrm{~cm}^{-1} ;{ }^{13} \mathrm{C}$ and ${ }^{1} \mathrm{H}$ NMR data, see Table 1; (+)-ESI-MS: $\mathrm{m} /$ $z 765.2[\mathrm{M}+\mathrm{H}]^{+}$; (-)-ESI-MS: $m / z 763.2[\mathrm{M}-\mathrm{H}]^{-}$; (+)-HR-ESIMS $m / z 765.1819[\mathrm{M}+\mathrm{H}]^{+}$(calcd. for $\mathrm{C}_{41} \mathrm{H}_{33} \mathrm{O}_{15}, 765.1819$ ), $787.1640[\mathrm{M}+\mathrm{Na}]^{+}$(calcd. for $\mathrm{C}_{41} \mathrm{H}_{32} \mathrm{O}_{15} \mathrm{Na}, 787.1639$ ).

\section{Cell Culture and Proliferation Inhibition Assay}

The human AML cell line MV4-11 (CRL-9591) was purchased from ATCC and cultured in IMDM (Gibco) supplemented with $10 \%$ FBS (Gibco) and 1\% penicillin-streptomycin (Gibco). To conduct cell proliferation assay, cells $\left(1.5 \times 10^{6}\right.$ cells/well $)$ in the logarithmic phase were seeded into 96 -well plates simultaneously with various concentrations of different compounds $(5 \mu \mathrm{l}$, final concentration of $50-0.023 \mu \mathrm{M}$ for $\mathrm{IC}_{50}$ determination) or vehicle (0.5\% DMSO) for $48 \mathrm{~h}$. Cell viability of compounds $\mathbf{1 - 4}$ was measured using Cell Counting Kit-8 (DoJINDO) according to the manufacturer's instructions with triptolide as positive control (Table 2). The absorbance was measured at $450 \mathrm{~nm}$ using a microplate reader (Epoch, Bio-Tek, United States). The value of half maximal inhibitory concentration $\left(\mathrm{IC}_{50}\right)$ was calculated using GraphPad Prism 7.

\section{Antimicrobial Assay}

Standard strains of Staphylococcus aureus (ATCC 29213), Escherichia coli (ATCC 25922), Bacillus subtilis (A186), Pseudomonas aeruginosa (ATCC 27853), and Acinetobacter baumannii (ATCC 19606) were obtained from CICC (China Center of Industrial Culture Collection, China). Bacteria were inoculated in LB Broth media and incubated overnight at $37^{\circ} \mathrm{C}$. The cultures were quantified via a spectrophotometer, and then diluted to $\mathrm{A}=0.02\left(\mathrm{OD}_{600}\right)$ and dispensed to 96-well black, clearbottom assay plates $(100 \mu \mathrm{l} /$ well). Test compounds (final concentration $50 \mu \mathrm{M}$ ) and controls (positive control of $50 \mu \mathrm{M}$ polymyxin and placebo control of DMSO) were then added. The plates were incubated for $16 \mathrm{~h}$ at $37^{\circ} \mathrm{C}$, and then measured the absorbance at $600 \mathrm{~nm}$ using a microplate reader. Compound activity was calculated on a per-plate basis (Zhao et al., 2020a).

\section{RESULTS AND DISCUSSION}

Preliminary HPLC-HRMS metabolic profiling of endophytic actinomycete strains isolated from D. huoshanense plant samples revealed that Streptomyces sp. HS-3-L-1 is capable of<smiles>CC(=O)c1c(O)cc(O)cc1-c1c(O)cccc1Cc1cc(O)c(Cc2c(O)cc(Cc3cccc(O)c3C(=O)c3c(C)cc(O)cc3O)oc2=O)c(=O)o1</smiles><smiles>[R][R]([H])O</smiles><smiles>Cc1cc(O)cc(O)c1C(=O)c1c(O)cccc1Cc1cc(O)cc(=O)o1</smiles><smiles>COc1cc(C)cc(C(=O)O)c1C1=C(O)C(=O)c2c(O)cccc2C1=O</smiles><smiles>COc1cc(/C=C/C(=O)NCCCCNC(=O)/C=C/c2ccc(O)c(OC)c2)ccc1O</smiles>

$\mathrm{HO}$<smiles>CC(=O)c1c(O)cc2c(c1C)C(=O)CC(C)C2</smiles>

7

FIGURE 1 | Chemical structures of compounds isolated from Streptomyces sp. HS-3-L-1. 

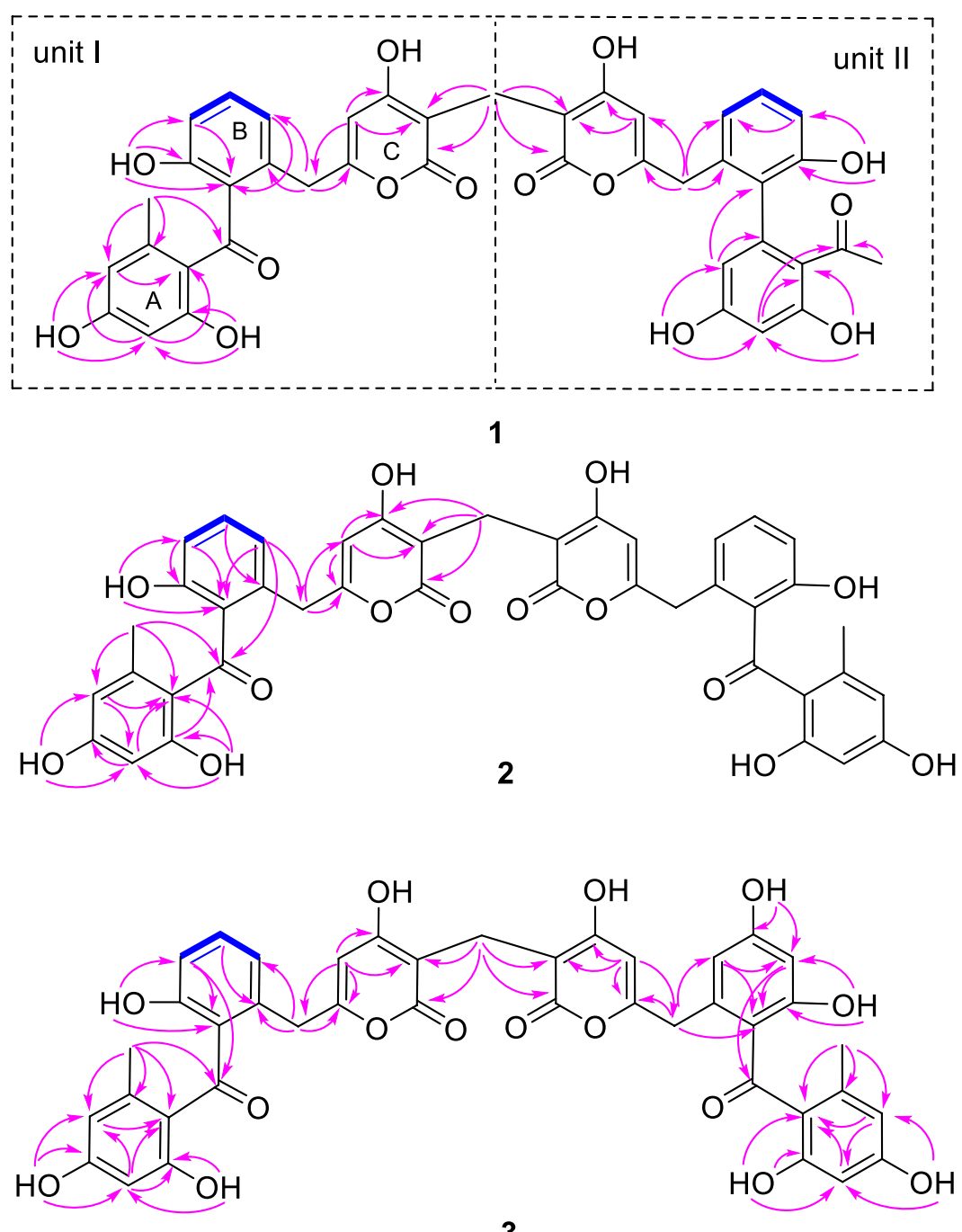

3

FIGURE 2 | Key COSY (bolds, blue), HMBC (arrows, pink) correlations of 1-3.

novel secondary metabolite production (Supplementary Figure S1). After scale-up fermentation (10 L) of Streptomyces sp. HS-3L-1, and further extraction, fractionation, and standard chromatography, three new polyketide dimers were isolated and identified [huoshanmycins A (1, yield: $1.68 \mathrm{mg} / \mathrm{L}), \mathrm{B}$ (2, yield: $0.86 \mathrm{mg} / \mathrm{L})$, and C (3, yield: $0.58 \mathrm{mg} / \mathrm{L})$ ], together with four previously reported metabolites, SEK43 (4, yield: $0.76 \mathrm{mg} / \mathrm{L})$, WS-5995 C (5, yield: $1.36 \mathrm{mg} / \mathrm{L})$, JBIR-94 (6, yield: $12.3 \mathrm{mg} / \mathrm{L})$, and GTRI-02 (7, yield: $0.94 \mathrm{mg} / \mathrm{L})$ ] (Figure 1).

Compound 1 was obtained as a yellow amorphous powder. Its molecular formula was established as $\mathrm{C}_{41} \mathrm{H}_{32} \mathrm{O}_{14}$ based on HRESIMS data, which indicated 26 degrees of unsaturation. The ${ }^{13} \mathrm{C}$ NMR of compound 1 showed 41 carbons, which can be sorted into two methyls, three methylenes, twelve aromatic methines, twenty $\mathrm{sp}^{2}$ quarternary carbons, and four carbonyls/ carboxylic acids, with the aid of HSQC spectrum. The ${ }^{1} \mathrm{H}-\mathrm{NMR}$ and HSQC spectra of 1 indicated twelve aromatic protons $\left[\delta_{\mathrm{H}}\right.$ $5.70(1 \mathrm{H}, \mathrm{s}), 5.76(1 \mathrm{H}, \mathrm{s}), 5.95(1 \mathrm{H}, \mathrm{d}, J=2.4 \mathrm{~Hz}), 6.09(1 \mathrm{H}, \mathrm{d}, J=$
$2.0 \mathrm{~Hz}), 6.13(1 \mathrm{H}, \mathrm{d}, J=2.3 \mathrm{~Hz}), 6.27(1 \mathrm{H}, \mathrm{d}, J=2.4 \mathrm{~Hz}), 6.74(2 \mathrm{H}$, $\mathrm{dd}, J=2.1,7.6 \mathrm{~Hz}), 6.80(2 \mathrm{H}, \mathrm{t}, J=8.7 \mathrm{~Hz}), 7.17(1 \mathrm{H}, \mathrm{t}, J=7.9 \mathrm{~Hz})$, and $7.22(1 \mathrm{H}, \mathrm{t}, J=7.7 \mathrm{~Hz})]$, two methyls $\left[\delta_{\mathrm{H}} 1.83\right.$ and 1.87 (each $3 \mathrm{H}, \mathrm{s})$ ], three methylenes $\left[\delta_{\mathrm{H}} 3.25,3.47\right.$, and 3.54 (each $\left.2 \mathrm{H}, \mathrm{s}\right)$ ], and six hydroxyls showed at low field: $\delta_{\mathrm{H}} 9.48,9.81,10.36,10.44$, 12.50 , and 12.66 (each $1 \mathrm{H}, \mathrm{s})$. Two spin systems of $\mathrm{H}-8\left(\delta_{\mathrm{H}} 6.74\right) /$ $\mathrm{H}-9\left(\delta_{\mathrm{H}} 7.22\right) / \mathrm{H}-10\left(\delta_{\mathrm{H}} 6.80\right)$ and $\mathrm{H}-8^{\prime}\left(\delta_{\mathrm{H}} 6.74\right) / \mathrm{H}-9^{\prime}\left(\delta_{\mathrm{H}} 7.17\right) /$ $\mathrm{H}-10^{\prime}\left(\delta_{\mathrm{H}} 6.80\right)$ observed in COSY spectrum suggested the presence of two 1,2,3-trisubstiuted benzene rings. Two aromatic protons $\delta_{\mathrm{H}} 6.13(\mathrm{H}-16)$ and $6.09(\mathrm{H}-18)$, together with the HMBC correlations from $\mathrm{H}_{3}-20\left(\delta_{\mathrm{H}} 1.83\right)$ to $\mathrm{C}-13$ $\left(\delta_{\mathrm{C}} 200.4\right), \mathrm{C}-18\left(\delta_{\mathrm{C}} 112.0\right)$, and $\mathrm{C}-19\left(\delta_{\mathrm{C}} 143.4\right)$, and from $\mathrm{H}-$ 16 to $\mathrm{C}-14\left(\delta_{\mathrm{C}} 116.0\right)$ and $\mathrm{C}-18$, and from $\mathrm{H}-18$ to C-14, C-16 $\left(\delta_{\mathrm{C}}\right.$ $101.1)$, and $\mathrm{C}-20\left(\delta_{\mathrm{C}} 21.9\right)$ suggested the presence of a 2,4dihydroxy-6-methyl-1-keto-phenyl moiety (ring $\mathrm{A}$ in Figure 2). The two active hydrogens showed key HMBC correlations from $15-\mathrm{OH}\left(\delta_{\mathrm{H}} 12.66, \mathrm{~s}\right)$ to $\mathrm{C}-14 / \mathrm{C}-15 / \mathrm{C}-16$, and from $17-\mathrm{OH}\left(\delta_{\mathrm{H}} 10.44, \mathrm{~s}\right)$ to $\mathrm{C}-16 / \mathrm{C}-17 / \mathrm{C}-18$, indicating two 
hydroxyl groups located at C-15 and C-17. An unsaturated sixmember lactone ring (ring $\mathrm{C}$ in Figure 2) was constructed by $\mathrm{HMBC}$ cross peaks from $\mathrm{H}-4\left(\delta_{\mathrm{H}} 5.76\right)$ to $\mathrm{C}-2\left(\delta_{\mathrm{C}} 100.0\right), \mathrm{C}-3\left(\delta_{\mathrm{C}}\right.$ $166.1)$, and $\mathrm{C}-6\left(\delta_{\mathrm{C}} 36.5\right)$, and from $\mathrm{H}-1^{\prime \prime}$ to $\mathrm{C}-1\left(\delta_{\mathrm{C}} 165.2\right)$ and $\mathrm{C}-$ 2. The obvious HMBC correlations from $\mathrm{H}_{2}-6\left(\delta_{\mathrm{H}} 3.54\right)$ to $\mathrm{C}-4$ $\left(\delta_{\mathrm{C}} 101.8\right), \mathrm{C}-5\left(\delta_{\mathrm{C}} 160.9\right), \mathrm{C}-7\left(\delta_{\mathrm{C}} 133.2\right)$ and $\mathrm{C}-8\left(\delta_{\mathrm{C}} 121.3\right)$ permitted the assembly of ring $\mathrm{B}$ and ring $\mathrm{C}$ through a $\mathrm{CH}_{2}$ linkage. Analysis of the remaining NMR data revealed that unit I of 1 (Figure 2) as C2-substitued SEK43 (4) (Meurer et al., 1997). SEK43 was reported as an engineered biosynthesis product, which was isolated and identified likewise from this endophytic strain. In a similar manner, the other decaketiderelated subunit II was elucidated as $\mathrm{C}^{\prime}$ '-substitued UMW4 (Meurer et al., 1997) mainly through HMBC correlations (Figure 2), e.g., from $\mathrm{H}-4^{\prime}\left(\delta_{\mathrm{H}} 5.70, \mathrm{~s}\right)$ to $\mathrm{C}-2^{\prime}\left(\delta_{\mathrm{C}} 100.1\right)$ and $\mathrm{C}-3^{\prime}\left(\delta_{\mathrm{C}} 166.3\right)$, from $\mathrm{H}_{2}-6^{\prime}\left(\delta_{\mathrm{H}} 3.47, \mathrm{~s}\right)$ to $\mathrm{C}-4^{\prime}, \mathrm{C}-5^{\prime}\left(\delta_{\mathrm{C}} 161.4\right)$, C-7 ${ }^{\prime}\left(\delta_{\mathrm{C}} 134.8\right)$, and C-8 $8^{\prime}\left(\delta_{\mathrm{C}} 120.9\right)$, from H-14 $4^{\prime}\left(\delta_{\mathrm{H}} 5.95, \mathrm{~d}\right)$ to C$12^{\prime}\left(\delta_{\mathrm{C}} 129.6\right)$ and $\mathrm{C}-13^{\prime}\left(\delta_{\mathrm{C}} 141.1\right)$, and from $\mathrm{H}-16^{\prime}\left(\delta_{\mathrm{H}} 6.27, \mathrm{~d}\right)$ to $\mathrm{C}-14^{\prime}\left(\delta_{\mathrm{C}} 111.3\right), \mathrm{C}-18^{\prime}\left(\delta_{\mathrm{C}} 115.9\right)$, and $\mathrm{C}-19^{\prime}\left(\delta_{\mathrm{C}} 203.6\right)$. The strong $\mathrm{HMBC}$ correlations from $\mathrm{H}_{2}-1^{\prime \prime}\left(\delta_{\mathrm{H}} 3.25\right)$ to $\mathrm{C}-1\left(\delta_{\mathrm{C}}\right.$ $165.2), \mathrm{C}-2\left(\delta_{\mathrm{C}} 100.0\right), \mathrm{C}-1^{\prime}\left(\delta_{\mathrm{C}} 161.9\right)$, and C-2 ${ }^{\prime}\left(\delta_{\mathrm{C}} 100.1\right)$ unambiguously connected SEK43 and UMW4 with a unique methylene bridge, to form the final dimeric structure of $\mathbf{1}$. Thus, compound $\mathbf{1}$ was identified as a novel polyketide dimer and named huoshanmycin A, to reflect the producing strain's point of origin.

Compound 2 was also obtained as a white amorphous powder and shared the same molecular formula $\left(\mathrm{C}_{41} \mathrm{H}_{32} \mathrm{O}_{14}\right)$ with huoshanmycin A (1). Compound 2 was clearly recognized as a polyketide dimer from its NMR data (Table 1), which showed only 21 carbons in ${ }^{13} \mathrm{C}$ NMR spectrum. Analysis of NMR data of compound 2 revealed that it was highly similar to SEK43 (4) and shared a same methylene linkage between $\mathrm{C}-2$ and $\mathrm{C}-2^{\prime}$. This was confirmed by $\mathrm{HMBC}$ correlations from $\mathrm{H}_{2}-1$ " $\left(\delta_{\mathrm{H}} 3.24\right)$ to C-1/C$1^{\prime}\left(\delta_{\mathrm{C}} 165.2\right), \mathrm{C}-2 / \mathrm{C}-2^{\prime}\left(\delta_{\mathrm{C}} 100.1\right)$, and C-3/C-3' $\left(\delta_{\mathrm{C}} 166.1\right)$. The remaining $\mathrm{HMBC}$ correlations (Figure 2) and NMR data (Table 1) were in full agreement with the new structure of compound 2, and it was named huoshanmycin B.

Compound 3 was obtained as a yellow amorphous powder and its molecular formula was determined as $\mathrm{C}_{41} \mathrm{H}_{32} \mathrm{O}_{15}$ from HRESIMS results. A detailed comparison of the NMR data of $\mathbf{3}$ and $\mathbf{2}$ indicated that their structures were highly similar. The significant differences observed in NMR spectra was that one of the 1,2,3-trisubstiuted benzene ring protons in $2\left(\delta_{\mathrm{H}} 6.75,7.22\right.$, $6.80)$ was replaced with two olefinic methines $\left(\delta_{\mathrm{H}} 6.16,6.23\right)$ and one hydroxyl $\left(\delta_{\mathrm{H}} 9.88\right)$ in compound 3 . This tetra-substituted benzene moiety was confirmed by the HMBC correlations from H-6 $6^{\prime}\left(\delta_{\mathrm{H}} 3.48\right)$ to $\mathrm{C}-7, \mathrm{C}-8^{\prime}$, and C- $12^{\prime}$, from $\mathrm{H}-8^{\prime}\left(\delta_{\mathrm{H}} 6.16\right)$ to C$6^{\prime}, \mathrm{C}-9^{\prime}, \mathrm{C}-10^{\prime}$, and $\mathrm{C}-12^{\prime}$, and from $\mathrm{H}-10^{\prime}\left(\delta_{\mathrm{H}} 6.23\right)$ to $\mathrm{C}-8^{\prime}, \mathrm{C}-9^{\prime}$, $\mathrm{C}-11^{\prime}$, and $\mathrm{C}-12^{\prime}$, as well as the correlation signals from $9^{\prime}-\mathrm{OH}$ $\left(\delta_{\mathrm{H}} 9.88\right)$ to $\mathrm{C}-8^{\prime}, \mathrm{C}-9^{\prime}$, and $\mathrm{C}-10^{\prime}$. Therefore, the structure of huoshanmycin C (3) was elucidated as shown in Figure 1.

The other five known compounds were identified as SEK43 (4) (McDaniel et al., 1995), WS-5995 C (5) (Ikushima et al., 1983), JBIR-94 (6) (Taj and Sorensen, 2015), and GTRI-02 (7) (Yeo et al., 1998), through comparison with reported data. Although compounds 1-7 were inactive at or below $50 \mu \mathrm{M}$ in a standard antimicrobial assay, huoshanmycins $A$ and $B(1,2)$, the two isomers, showed antiproliferative activity against the MV4-11 cell line with $\mathrm{IC}_{50}$ values of $32.9 \pm 7.2$ and $33.2 \pm 6.1 \mu \mathrm{M}$, respectively (Table 2).

In summary, the discovery of compounds 1-7 as metabolites of the D. huoshanense isolate Streptomyces sp. HS-3-L-1 further highlights the potential for novel microbial natural product discovery from medicinal plants. Among them, compounds 1-3 were unique dimers of SEK43 (4), SEK15, or UWM4, three decaketide-related shunt products discovered from minimal jadPKS constructs (Meurer et al., 1997). So far, only two similar natural products, strepolyketides B and C, were recently reported from a marine-derived Streptomyces (Jiang et al., 2020). Moreover, huoshanmycins A and B showed moderate cytotoxicity against MV4-11 human leukemia cell. The newly isolated 1-3 enriched the structural diversity of microbial source. Future investigation to explore the biosynthetic logic of these structurally unique dimers is ongoing.

\section{DATA AVAILABILITY STATEMENT}

The datasets presented in this study can be found in online repositories. The names of the repository/repositories and accession number(s) can be found below: https://www.ncbi. nlm.nih.gov/, OK161010.

\section{AUTHOR CONTRIBUTIONS}

YZ contributed to the chemical experiments and prepared the manuscript draft. YK performed the identification of strain and the cytotoxicity assay. $\mathrm{YuH}, \mathrm{LZ}$, and SL participated in the endophytic strain isolation and antimicrobial assay. $\mathrm{SH}$ and XC undertook the MS analysis. TX contributed to collection of plant samples and financial support. $\mathrm{YaH}$ and XW contributed to the design of experiment, financial support, and manuscript revision. All authors have approved the submission of this manuscript for publication.

\section{FUNDING}

This research was financially supported by the Jiangsu Provincial Innovation and Entrepreneurship Talent Plan (202010528), the Nature Science Foundation of Jiangsu Higher Education Institutions of China (No. 19KJA180008), the National Natural Science Foundation (81803391), and the Natural Science Foundation Youth Program of Nanjing University of Chinese Medicine (NZY81803391).

\section{SUPPLEMENTARY MATERIAL}

The Supplementary Material for this article can be found online at: https://www.frontiersin.org/articles/10.3389/fchem.2021.807508/ full\#supplementary-material 


\section{REFERENCES}

Chen, S., Dai, J., Song, X., Jiang, X., Zhao, Q., Sun, C., et al. (2020). Endophytic Microbiota Comparison of Dendrobium huoshanense Root and Stem in Different Growth Years. Planta Med. 86, 967-975. doi:10.1055/a-1046-1022

Chen, S. T., Dai, J., Jiang, X. P., Song, X. W., Chen, C. W., Chen, N. F., et al. (2019). Diversity and Difference of Endophytes in Dendrobium huoshanense with Different Growth Years. Zhongguo Zhong Yao Za Zhi 44, 1145-1150. doi:10.19540/j.cnki.cjcmm.2019.0022

Chen, X., Hu, J., Wu, S., Zhao, H., Peng, D., Wu, D., et al. (2022). Cytotoxic picrotoxane-type sesquiterpenoid lactones from Dendrobium huoshanense. Rec. Nat. Prod. 16, 144-149. doi:10.25135/rnp.260.21.04.2048

Cui, Y., Yi, D., Bai, X., Sun, B., Zhao, Y., and Zhang, Y. (2012). Ginkgolide B Produced Endophytic Fungus (Fusarium oxysporum) Isolated from Ginkgo Biloba. Fitoterapia 83, 913-920. doi:10.1016/j.fitote.2012.04.009

Ding, N., Wang, J., Liu, J., Zhu, Y., Hou, S., Zhao, H., et al. (2021). Cytotoxic Guaianolide Sesquiterpenoids from Ainsliaea fragrans. J. Nat. Prod. 84, 2568-2574. doi:10.1021/acs.jnatprod.1c00587

Fan, X., Han, J., Zhu, L., Chen, Z., Li, J., Gu, Y., et al. (2020). Protective activities of Dendrobium huoshanense C. Z. Tang et S. J. Cheng polysaccharide against highcholesterol diet-induced atherosclerosis in zebrafish. Oxidative Med. Cell Longevity 2020, 1-10. doi:10.1155/2020/8365056

Ge, J.-C., Zha, X.-Q., Nie, C.-Y., Yu, N.-J., Li, Q.-M., Peng, D.-Y., et al. (2018). Polysaccharides from Dendrobium huoshanense Stems Alleviates Lung Inflammation in Cigarette Smoke-Induced Mice. Carbohydr. Polym. 189, 289-295. doi:10.1016/j.carbpol.2018.02.054

Gómez, O. C., and Luiz, J. H. H. (2018). Endophytic Fungi Isolated from Medicinal Plants: Future Prospects of Bioactive Natural Products from Tabebuia/ Handroanthus Endophytes. Appl. Microbiol. Biotechnol. 102, 9105-9119. doi:10.1007/s00253-018-9344-3

Hu, Y., Zhao, H., Yang, A., Lv, Q., Ding, N., Lu, T.-L., et al. (2021). Jatrophacine, a 4,5-seco-Rhamnofolane Diterpenoid with Potent Anti-inflammatory Activity from Jatropha curcas. Nat. Product. Res. 35, 2748-2752. doi:10.1080/14786419. 2019.1660656

Ikushima, H., Takase, S., Kawai, Y., Itoh, Y., Okamoto, M., and Tanaka, H. (1983). Structure and Synthesis of New Anticoccidial Antibiotics Isolated from Streptomyces auranticolor. Agric. Biol. Chem. 47, 2231-2235. doi:10.1080/ 00021369.1983.10865932

Jiang, Y., Huang, Y., Chen, S., Ji, Y., Ding, W., and Ma, Z. (2020). Strepolyketides A-C, Three Novel SEK15-Derived Polyketides from Streptomyces sp. HN2A53. Tetrahedron Lett. 61, 151996. doi:10.1016/j.tetlet.2020.151996

Li, O., Xiao, R., Sun, L., Guan, C., Kong, D., and Hu, X. (2017). Bacterial and Diazotrophic Diversities of Endophytes in Dendrobium catenatum Determined through Barcoded Pyrosequencing. PLoS One 12, e0184717. doi:10.1371/ journal.pone.0184717

Li, Q.-M., Jiang, H., Zha, X.-Q., Wu, D.-L., Pan, L.-H., Duan, J., et al. (2020). Antiinflammatory Bibenzyls from the Stems of Dendrobium huoshanense via Bioassay Guided Isolation. Nat. Product. Res. 34, 563-566. doi:10.1080/ 14786419.2018.1489394

McDaniel, R., Ebert-Khosla, S., Hopwood, D. A., and Khosla, C. (1995). Rational Design of Aromatic Polyketide Natural Products by Recombinant Assembly of Enzymatic Subunits. Nature 375, 549-554. doi:10.1038/375549a0

Meurer, G., Gerlitz, M., Wendt-Pienkowski, E., Vining, L. C., Rohr, J., and Richard Hutchinson, C. (1997). Iterative Type II Polyketide Synthases, Cyclases and Ketoreductases Exhibit Context-dependent Behavior in the Biosynthesis of Linear and Angular Decapolyketides. Chem. Biol. 4, 433-443. doi:10.1016/ s1074-5521(97)90195-2

Newman, D. J., and Cragg, G. M. (2015). Endophytic and Epiphytic Microbes as sources of Bioactive Agents. Front. Chem. 3, 34. doi:10.3389/fchem.2015.00034

Raja, A., and Prabakaran, P. (2011). Actinomycetes and Drug-An Overview. Am. J. Drug Discov. Develop. 1, 75-84. doi:10.3923/ajdd.2011.75.84

Stierle, A., Strobel, G., and Stierle, D. (1993). Taxol and Taxane Production by Taxomyces Andreanae, an Endophytic Fungus of Pacific Yew. Science 260, 214-216. doi:10.1126/science. 8097061
Taj, R., and Sorensen, J. L. (2015). Synthesis of Actinomycetes Natural Products JBIR-94, JBIR-125, and Related Analogues. Tetrahedron Lett. 56, 7108-7111. doi:10.1016/j.tetlet.2015.11.020

Tian, C.-C., Zha, X.-Q., Pan, L.-H., and Luo, J.-P. (2013). Structural Characterization and Antioxidant Activity of a Low-Molecular Polysaccharide from Dendrobium huoshanense. Fitoterapia 91, 247-255. doi:10.1016/j.fitote.2013.09.018

Tsavkelova, E. A., Cherdyntseva, T. A., Klimova, S. Y., Shestakov, A. I., Botina, S. G., and Netrusov, A. I. (2007). Orchid-associated Bacteria Produce Indole-3Acetic Acid, Promote Seed Germination, and Increase Their Microbial Yield in Response to Exogenous Auxin. Arch. Microbiol. 188, 655-664. doi:10.1007/ s00203-007-0286-X

Wang, H.-Y., Li, Q.-M., Yu, N.-J., Chen, W.-D., Zha, X.-Q., Wu, D.-L., et al. (2019). Dendrobium huoshanense Polysaccharide Regulates Hepatic Glucose Homeostasis and Pancreatic $\beta$-cell Function in Type 2 Diabetic Mice. Carbohydr. Polym. 211, 39-48. doi:10.1016/j.carbpol.2019.01.101

Wang, X.-C., Zheng, Z.-P., Gan, X.-W., and Hu, L.-H. (2009). Jatrophalactam, a Novel Diterpenoid Lactam Isolated from Jatropha curcas. Org. Lett. 11, 5522-5524. doi:10.1021/ol902349f

Wang, X., Shaaban, K. A., Elshahawi, S. I., Ponomareva, L. V., Sunkara, M., Zhang, Y., et al. (2013). Frenolicins C-G, Pyranonaphthoquinones from streptomyces sp. RM-4-15. J. Nat. Prod. 76, 1441-1447. doi:10.1021/ np400231r

Wang, Y.-H. (2021). Traditional Uses, Chemical Constituents, Pharmacological Activities, and Toxicological Effects of Dendrobium Leaves: A Review. J. Ethnopharmacology 270, 113851. doi:10.1016/j.jep.2021.113851

Xu, F., Chen, X., Hu, J., Zhao, H., Peng, D., Wu, D., et al. (2022). Cytotoxic Picrotoxane-type Sesquiterpenoid Lactones from Dendrobium huoshanense. Rec. Nat. Prod. 16, 144-149. doi:10.25135/rnp.260.21.04. 2048

Yeo, W.-H., Yun, B.-S., Kim, S.-S., Park, E.-K., Kim, Y.-H., Yoo, I.-D., et al. (1998) GTRI-02, a New Lipid Peroxidation Inhibitor from Micromonospora sp. SA246. J. Antibiot. 51, 952-953. doi:10.7164/antibiotics.51.952

Zhao, H., Chen, X., Chen, X., Zhu, Y., Kong, Y., Zhang, S., et al. (2020c). New Peptidendrocins and Anticancer Chartreusin from an Endophytic Bacterium of Dendrobium officinale. Ann. Transl. Med. 8, 455. doi:10.21037/atm.2020. 03.227

Zhao, H., Yang, A., Liu, J., Bao, S., Peng, R., Hu, Y., et al. (2020b). Chartspiroton, a Tetracyclic spiro-naphthoquinone Derivative from a Medicinal Plant Endophytic Streptomyces. Org. Lett. 22, 3739-3743. doi:10.1021/acs.orglett. 0c00696

Zhao, H., Yang, A., Zhang, N., Li, S., Yuan, T., Ding, N., et al. (2020a). Insecticidal Endostemonines A-J Produced by Endophytic Streptomyces from Stemona sessilifolia. J. Agric. Food Chem. 68, 1588-1595. doi:10.1021/acs.jafc.9b06755

Zhu, Y., Li, S., Kong, Y., Zhao, H., Hu, Y., Meng, J., et al. (2021). Terragines F-G Produced by Endophytic bacillus sp. SH-1.2-ROOT-18 from Dendrobium officinale. Nat. Product. Res. doi:10.1080/14786419.2021.1914614

Conflict of Interest: The authors declare that the research was conducted in the absence of any commercial or financial relationships that could be construed as a potential conflict of interest.

Publisher's Note: All claims expressed in this article are solely those of the authors and do not necessarily represent those of their affiliated organizations, or those of the publisher, the editors, and the reviewers. Any product that may be evaluated in this article, or claim that may be made by its manufacturer, is not guaranteed or endorsed by the publisher.

Copyright (c) 2022 Zhu, Kong, Hong, Zhang, Li, Hou, Chen, Xie, Hu and Wang. This is an open-access article distributed under the terms of the Creative Commons Attribution License (CC BY). The use, distribution or reproduction in other forums is permitted, provided the original author(s) and the copyright owner(s) are credited and that the original publication in this journal is cited, in accordance with accepted academic practice. No use, distribution or reproduction is permitted which does not comply with these terms 